\title{
Análise da energia eólica no Brasil usando Séries Temporais
}

\author{
Analysis of wind energy in Brazill using Time Series \\ Análisis de la energía eólica en Brasil mediante Series Temporales
}

Recebido: 16/12/2021 | Revisado: 22/12/2021 | Aceito: 28/12/2021 | Publicado: 06/01/2022

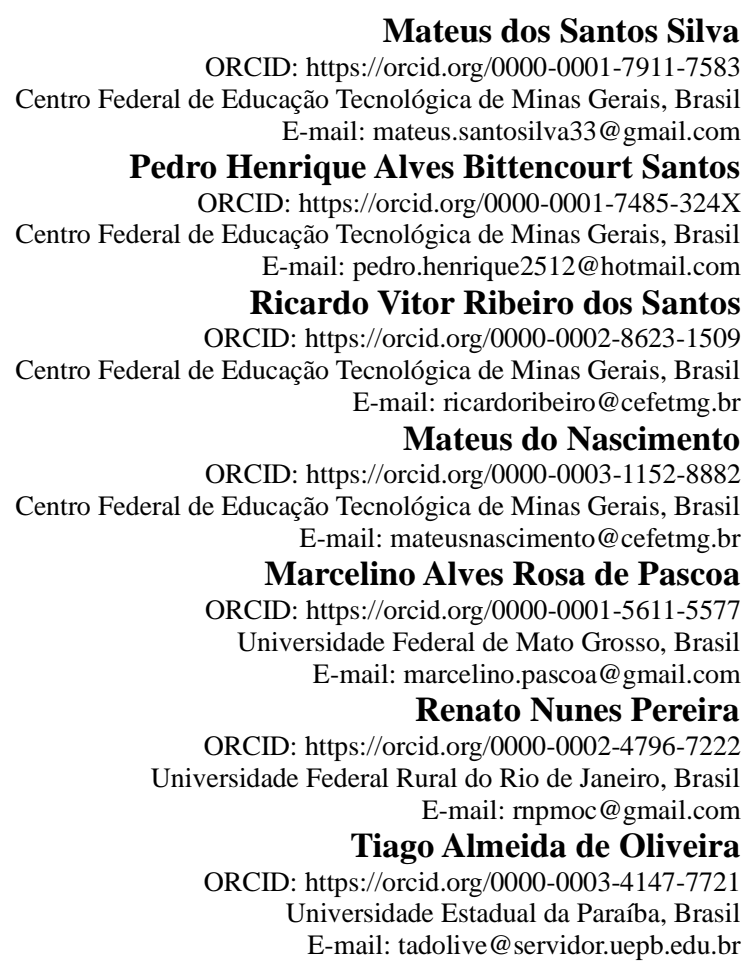

\section{Resumo}

O objetivo deste trabalho foi ajustar um modelo de séries temporais à série de energia elétrica gerada pela matriz eólica com a finalidade de estudar a presença de tendência, sazonalidade e realizar previsões. A série histórica utilizada consiste da produção de energia elétrica gerada pela matriz eólica, coletada mensalmente pelo Operador Nacional do Sistema Elétrico. A série está compreendida entre jan/2007 a mar/2021, com 171 observações. A série foi dividida em dois subconjuntos, ao primeiro (jan/2007 a dez/2019) foi designado o processo de modelagem (calibração) e ao segundo (jan/2020 a mar/2021) foi atribuído a avaliação das previsões (validação). O horizonte de previsão contemplou o período de abr/2021 a dez/2022. Realizou-se uma transformação Box-Cox na escala dos dados para tornar o modelo aditivo. Verificou-se a presença da componente de tendência. A partir dos correlogramas da FAC e FACP da série original transformada com uma diferença de ordem 1 foi possível propor alguns modelos. Buscaram-se modelos com resíduos não correlacionados e com menor AIC. Destes, realizaram-se previsões para o período de jan/2020 a mar/21 que foram comparadas com observações reais através do EQMP. O modelo SARIMA $(5,1,2) \times(0,0,3)_{12}$ foi escolhido, pois apresentou o menor EQMP. Observou-se que os meses subsequentes continuam seguindo o padrão crescente que ela vem mantendo desde de 2015. O modelo proposto para prever a quantidade de energia elétrica gerada pela matriz eólica de curto prazo vai permitir que os gestores tenham tempo suficiente para programar a operação de distribuição de energia de forma adequada.

Palavras-chave: Energia eólica; Tendência; Previsões; SARIMA.

\begin{abstract}
This paper aim was, adjust a time series model to the generated electrical energy series through the wind grid for studying trend, seasonality and making predictions. The used historic series consists in the electric energy generated through the wind grid, collected monthly by the Operador Nacional do Sistema Elétrico. The range is between jan/2007 until mar/2021, with 171 observations. The series was divides into two groups, first (jan/2007 to dec/2019) used for modelling process (calibration) and the other one (jan/2020 to mar/2021) for predictions evaluation (validation). For making predictions it was used apr/2021 to dec/2022 period. In the procedures, first was applied a Box-Cox transform on data scale for turn the model into additive. Then, the presence of trend was checked. From the
\end{abstract}


transformed original series with an order 1 difference, FAC and FACP correlograms, were possible purpose some models. The residual not correlated and a lower AIC were the criteria used for the models. From the chose ones, were made prediction for jan/2020 to mar/2021 period, that were compared to the real observations through EQMP. The SARIMA $(5,1,2) \times(0,0,3) 12$ model was chosen because of its lowest EQMP. Other observation is related to the next months followed a rising pattern since 2015. The purposed model for forecasting the amount of electric energy generated by the wind grid will help the managers, giving them time for programming the proper energy's distribution.

Keywords: Wind energy; Trend; Forecasts; SARIMA.

\section{Resumen}

El objetivo de este trabajo fue ajustar un modelo de series temporales a la serie de energía eléctrica generada a través de la red eólica para estudiar la tendencia, la estacionalidad y realizar predicciones. La serie histórica utilizada consiste en la energía eléctrica generada a través de la red eólica, recogida mensualmente por el Operador Nacional do Sistema Elétrico. El intervalo está comprendido entre enero/2007 y marzo/2021, con 171 observaciones. La serie fue dividida en dos grupos, el primero (ene/2007 a dic/2019) utilizado para el proceso de modelización (calibración) y el otro (ene/2020 a mar/2021) para la evaluación de las predicciones (validación). Para realizar las predicciones se utilizó el periodo de abr/2021 a dic/2022. En los procedimientos, primero se aplicó una transformación Box-Cox en la escala de datos para convertir el modelo en aditivo. Luego, se verificó la presencia de la tendencia. A partir de la serie original transformada con una diferencia de orden 1, los correlogramas FAC y FACP, fueron posibles para algunos modelos. El residuo no correlacionado y un AIC menor fueron los criterios utilizados para los modelos. A partir de los elegidos, se realizaron predicciones para el periodo enero/2020 a marzo/2021, que fueron comparadas con las observaciones reales a través de EQMP. Se eligió el modelo SARIMA $(5,1,2) \times(0,0,3)_{12}$ por su menor EQMP. Otra observación está relacionada con que los próximos meses han seguido un patrón ascendente desde 2015. El modelo propuesto para predecir la cantidad de energía eléctrica generada por la red eólica ayudará a los gestores, dándoles tiempo para programar la distribución adecuada de la energia.

Palabras clave: Energía eólica; Tendencia; Previsiones; SARIMA.

\section{Introdução}

O aumento na demanda por energia elétrica no Brasil vem gerando uma preocupação nos setores públicos e privados. Em 2014, o Operador Nacional do Sistema Elétrico (ONS) apontou que o consumo de energia elétrica quebrou um novo recorde instantâneo de eletricidade, no valor de 85.708 megawatts (MW). De acordo com o boletim mensal de energia do Ministério de Minas e Energia (MME, 2021), o consumo de energia elétrica cresceu 6,9\% de janeiro a maio de 2021 com relação ao mesmo período de 2020.

As usinas hidrelétricas (UHE) reproduzem 62,8\% da potência instalada na matriz elétrica nacional, a geração das usinas termelétricas (UTE) caracteriza $28,2 \%$ da eletricidade instalada e as demais eólica, pequenas centrais hidrelétricas (PCH), centrais geradoras hidrelétricas (CGH) e solar, tem baixa contribuição na matriz elétrica brasileira (Alencar, 2018). As termelétricas tem a desvantagem de utilizar combustíveis fósseis como carvão, gás e petróleo, recursos estes que são finitos e não bastando isso, ainda são combustíveis caros e prejudiciais ao meio ambiente.

Segundo a Agência Nacional de Energia Elétrica (ANEEL), o Brasil possui um grande potencial para gerar energia elétrica através da matriz eólica, estimado em 143 gigawatts $(\mathrm{GW})$, com destaque para as regiões litorâneas do norte e nordeste, litoral sul do Rio Grande do Sul, sudeste do Paraná e vale do Rio São Francisco (Morais, 2015). A justificativa se deve ao fato de que o Brasil possui um volume de ventos duas vezes maior que a média mundial e baixa oscilação da velocidade, o que garante maior previsibilidade à geração de eletricidade.

Vale destacar que o potencial de energia instalado atualmente no Brasil, acrescentando todas as fontes de energia, gira em torno de $131 \mathrm{GW}$, portanto bem abaixo do potencial eólico estimado. No entanto, a participação da energia eólica na geração de energia elétrica apresenta um crescimento muito lento, mesmo com a criação do Programa de Incentivo às Fontes Alternativas de Energia Elétrica (PROINFA) em 2002, ainda assim, o Brasil ocupava a 9a posição entre os países que mais geram energia eólica no mundo (Braz et al., 2017).

Há alguns anos, as tecnologias desenvolvidas para operar parques eólicos tinham um custo muito superior às das 
hidrelétricas, porém com o progresso tecnológico e o benefício comercial, esses dois tipos de matrizes energéticas tem custos bem semelhantes. Nesse sentido, como o país tem um potencial considerado relevante para energia eólica, é imprescindível que ele direcione seus investimentos para uma expansão acentuada na produção de energia elétrica nesta área, uma vez que, o desenvolvimento sustentável é o tema que vem sendo muito discutido pela Organização das Nações Unidas (ONU).

Diversos estudos vêm aplicando técnicas estatísticas para estudar séries históricas sobre temas relacionados à energia eólica em diferentes regiões do país, dentre eles, destacam-se alguns. Alencar (2018) desenvolveu um modelo de previsão da velocidade do vento para geração de energia eólica utilizando metodologia baseada em um sistema híbrido, composto pelo modelo SARIMA e por redes neurais artificiais. Fernandes (2018) realizou a previsão de potência eólica de curto prazo baseada na análise espectral e decomposição da série temporal. Silva (2018) analisou e comparou a aplicação de três métodos de redução de dimensionalidade de séries temporais para análise dos valores extremos dessas séries de vento. Silva (2017) realizou análise e previsão de curto prazo do vento através de modelagem estatística em áreas de potencial eólico no Nordeste do Brasil. Malta (2009) ajustou um modelo de séries temporais para prever a velocidade dos ventos, a fim de avaliar a viabilidade de instalação de usinas eólicas.

Diante do exposto, o objetivo deste trabalho foi ajustar um modelo de séries temporais à série de energia elétrica gerada pela matriz eólica com a finalidade de estudar a presença de tendência, sazonalidade e realizar previsões.

Devido ao comportamento aleatório do vento e suas grandes oscilações, principalmente, em curtos períodos de tempo, faz-se necessário criar um sistema de previsão de curto e de longo prazo para prever a quantidade de energia elétrica gerada pela matriz eólica com o intuito de se ter um bom planejamento na operação, pois uma das maiores dificuldades, em comparação à previsão de energia de fontes convencionais, é lidar com a volatilidade do vento.

\section{Metodologia}

A série temporal utilizada neste artigo constitui a série histórica da produção de energia elétrica gerada pela matriz eólica coletada mensalmente pelo Operador Nacional do Sistema Elétrico (ONS, 2021). Esta série contém 171 observações mensais, dadas em megawatt-hora (MWh), compreendida entre janeiro de 2007 a marco de 2021. A série foi dividida em dois subconjuntos. Ao primeiro (período de jan/2007 a dez/2019) foi designado o processo de modelagem (calibração). Ao segundo (período de jan/2020 a mar/2021) foi atribuído a avaliação das previsões (validação). O horizonte de previsão contemplou o período de abr/2021 a dez/2022.

Uma série temporal é uma sequência de observações em intervalos de tempo regularmente espaçados. É a realização de um processo estocástico considerado como uma família de variáveis aleatórias supostamente definidas em um mesmo espaço de probabilidades, isto é, $Y=\left\{Y_{t}, t \in T\right\}$, sendo $T$ um conjunto qualquer e para cada $t \in T, Y_{t}$ é uma variável aleatória (Morettin \& Toloi, 2006).

Este estudo trata-se de uma pesquisa exploratória, de natureza quantitativa, uma vez que técnicas estatísticas foram utilizadas para análise e modelagem de dados, como descrito por Pereira, Shitsuka, Parreira, e Shitsuka (2018).

De início, plotou-se o gráfico da série original para averiguar a presença de possíveis componentes de tendência e/ou sazonalidade. Em seguida, a questão foi decidir qual modelo de equação que vai relacionar as componentes com a variável, modelo aditivo $\left(Y_{t}=T_{t}+S_{t}+\epsilon_{t}\right)$ ou modelo multiplicativo $\left(Y_{t}=T_{t} \times S_{t} \times \epsilon_{t}\right)$, onde $Y_{t}$ é o valor da série temporal, $T_{t}$ é a componente de tendência, $S_{t}$ é a componente de sazonalidade e $\epsilon_{t}$ é o componente aleatório, ambos no período $t$. Se o modelo for aditivo, a amplitude do padrão sazonal não se altera em torno da tendência quando esta aumenta ou diminui ao longo do tempo, no entanto, se a amplitude do padrão sazonal se alterar em torno da tendência ao longo do tempo fazendo com que a variância não seja constante, tem-se o modelo multiplicativo. Neste caso, uma transformação Box-Cox (ver equação 1) 
na escala dos dados é necessária para estabilizar a variância e tornar o efeito sazonal aditivo. Uma forma de verificar a necessidade da transformação é construir um gráfico da amplitude versus média, onde no eixo das abscissas traça-se as médias das observações de subgrupos da série e no eixo das ordenadas, a amplitude desses subgrupos. Se a amplitude independer da média, ou seja, os pontos ficarem espalhados ao redor de uma reta paralela ao eixo horizontal, não se deve fazer a transformação nos dados.

$$
Y_{t}^{*}(\lambda)=\left\{\begin{array}{l}
\frac{\left(Y_{t}^{\lambda}-1\right)}{\lambda}, \lambda \neq 0 \\
\log Y_{t}, \lambda=0
\end{array}\right.
$$

Uma abordagem bastante aplicada na confecção de modelos paramétricos para séries temporais univariadas é conhecida como metodologia de Box-Jenkins. Para seu uso, é necessário que a série seja estacionária, ou melhor, que ela se propague aleatoriamente no tempo ao redor de uma média e variância constante (Morettin \& Toloi, 2006). Contudo, a maioria das séries apresenta componentes que tem uma forma de não estacionariedade, por exemplo, tendência (pode ser visualizada como um acréscimo ou um decréscimo sucessivo das observações no decorrer de um período) e/ou sazonalidade (são variações ocasionadas em períodos inferiores a um ano, ocorrendo de forma mensal, bimestral, trimestral, quadrimestral, semestral, etc).

Para investigar a presença das componentes de tendência e/ou sazonalidade utilizaram-se os testes de Cox-Stuart e Kruskal-Wallis, respectivamente (Santos et al., 2021). Caso a série apresente alguma das componentes citadas, o procedimento comum é tomar diferenças sucessivas na série original para torná-la estacionária. Para retirar a componente de tendência, tome a primeira diferença, definida por $\Delta Y_{t}=Y_{t}-Y_{t-1} \operatorname{com} t=1,2, \cdots, n$. De modo geral, duas diferenças bastam. A sazonalidade determinística pode ser removida através de uma diferença realizada na série por meio do método de médias móveis, considerando a ordem da variação ocorrida no período s.

Retiradas as componentes de tendência e/ou sazonalidade, a série atinge a estacionariedade e segue-se então para a identificação do modelo. Para propor um modelo é fundamental explorar as funções de autocorrelação FAC e autocorrelação parcial (FACP) da série diferenciada. As correlações significativas da FAC sugerem a ordem "q" do modelo de médias móveis (MA) e as da FACP, a ordem "p" do modelo autorregressivo (AR). No caso de haver correlações significativas nas defasagens múltiplas de período sazonal s, é necessário agregar a parte sazonal ao modelo.

Box et al., (2015) propuseram o modelo SARIMA (p,d,q)×(P,D,Q)s expresso pela equação:

$$
\phi(B) \Phi\left(B^{s}\right)(1-B)^{d}\left(1-B^{s}\right)^{D} Z_{t}=\theta(B) \theta\left(B^{s}\right) \epsilon_{t}
$$

em que (p,d,q) são as ordens do modelo referente à dinâmica ordinal e (P, D, Q) são as ordens da parte sazonal. Os polinômios autorregressivos e de médias móveis são definidos pelas seguintes equações:

$$
\begin{aligned}
& \phi(B)=1-\phi_{1} B-\cdots-\phi_{p} B^{p}(\text { ordem } \mathrm{p}) \\
& \theta(B)=1-\theta_{1} B-\cdots-\theta_{p} B^{q}(\text { ordem } \mathrm{q}) \\
& \Phi\left(B^{s}\right)=1-\Phi_{1} B^{s}-\cdots-\Phi_{p} B^{P_{s}}(\text { ordem } \mathrm{P}) \\
& \left.\theta\left(B^{s}\right)=1-\Theta_{1} B^{s}-\cdots-\theta_{Q} B^{Q s} \text { (ordem } \mathrm{Q}\right)
\end{aligned}
$$

Os operadores diferença são $(1-B)^{d} \mathrm{e}\left(1-B^{S}\right)^{D}$, sendo $d$ o número de diferenças necessárias para remover a 
tendência e $D$ o número de diferenças de defasagens s necessárias para remover a sazonalidade;

$Z_{t}$ representa os valores da série original depois de efetuar as diferenças de ordem 1 e s, essenciais para torná-la estacionária; $\epsilon_{t}$ é o componente aleatório no tempo $t$.

Após a identificação dos possíveis modelos, com base nas defasagens significativas dos correlogramas das funções de autocorrelação e autocorrelação parcial, o próximo passo é estimar os parâmetros através do método de máxima verossimilhança.

Em seguida, realizou-se a análise de diagnóstico para verificar a adequabilidade dos modelos ajustados, isto é, certificar-se de que os resíduos apresentam normalidade e que eles não são correlacionados, ou seja, resíduos denominados de ruído branco. A normalidade dos resíduos foi confirmada pelo teste Shapiro-Wilk (Shapiro \& Wilk, 1965) e a ausência de autocorrelação serial foi validada pelo teste estatístico de Ljung-Box (Ljung \& Box, 1978).

Após o resultado da análise diagnóstica, selecionaram-se aqueles modelos em que os resíduos foram considerados ruído branco e apresentaram menores valores de medida de qualidade de ajuste para o critério de informação de Akaike - AIC (Delfino, Santos, \& Santos, 2021).

Com alguns modelos selecionados e ajustados, realizaram-se as previsões para a produção de energia elétrica gerada pela matriz eólica para o período de jan/2020 a mar/2021.

A previsão $Y_{t+h}$ denotada por $\hat{Y}_{t}(h)$ é a esperança matemática condicional de $Y_{t+h}$, dado $Y_{t}, Y_{t-1},{ }^{\cdots}$, ou melhor dizendo,

$$
\hat{Y}_{t}(h)=\left[Y_{t+h}\right]=E\left[Y_{t+h} \vee Y_{t}, Y_{t-1}, \cdots\right]
$$

Como uma das finalidades deste trabalho é fazer previsões, validou-se o modelo comparando as previsões obtidas com as observações da série original do segundo subconjunto por meio de outra medida de qualidade de ajuste, denominada de erro quadrático médio de previsão EQMP.

As estimativas do erro quadrático médio de previsão foram dadas pela média dos quadrados das diferenças entre valores observados e previstos. Logo, o EQMP com origem em $t$ é denotado por:

$$
E Q M P_{t}=\frac{1}{n} \sum_{h=1}^{n}\left[Y_{t+h}-\hat{Y}_{t}(h)\right]^{2}
$$

O modelo escolhido para descrever a série temporal e realizar o horizonte de previsão no período de abr/2021 a dez/2022 foi aquele que forneceu o menor erro quadrático médio de previsão no período de jan/2020 a mar/2021.

Foi utilizado o software $R$ (R Core Team, 2021) para realizar as análises, assim como os pacotes forecast (Hyndman et al., 2019) e tseries (Trapletti \& Hornik, 2021). O nível de significância considerado foi $\alpha=5 \%$.

\section{Resultados e Discussão}

A Figura 1 apresenta o gráfico da série temporal de energia elétrica gerada pela matriz eólica, em megawatt-hora (MWh), produzido mensalmente pelo Brasil no período de jan/2007 a dez/2019. Observa-se uma tendência expressiva na geração de energia a partir de 2015, ano em que o Banco Nacional de Desenvolvimento Econômico e Social (BNDES) liberou R \$ 6,6 bilhões para projetos de geração de energia eólica. Percebe-se também um comportamento periódico indicando um provável efeito sazonal. Sabe-se que não é possível confirmar essas afirmações sobre as componentes de tendência e/ou 
sazonalidade visualmente, para isso é necessário aplicar os testes Cox-Stuart e Kruskal-Wallis, respectivamente.

Figura 1: Série mensal da produção de energia elétrica gerada pela matriz eólica no período de jan/2007 a dez/2019.

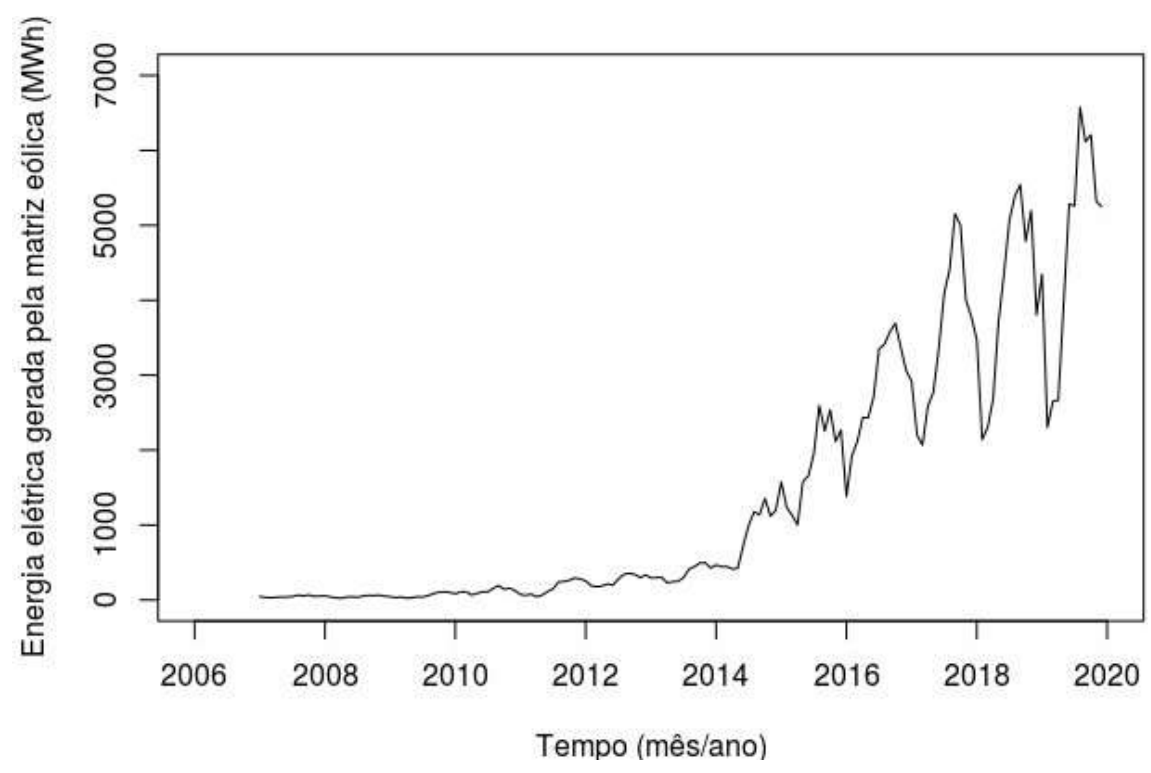

Fonte: Autores.

Antes de inferir sobre as componentes supracitadas, é necessário verificar se o modelo é aditivo ou multiplicativo, confeccionou-se então o gráfico da amplitude versus média, ver Figura 2. Através desse gráfico constatou-se uma relação positiva entre as médias das observações de subgrupos da série original e a amplitude desses subgrupos, com coeficiente de inclinação de 0,8715, estimado por meio de uma regressão linear simples. Foi aplicado o teste t para aferir estatisticamente, se o coeficiente angular da reta ajustado era significativo. Como o p-valor encontrado foi menor que 0,01, rejeitou-se a hipótese nula de que o coeficiente angular da reta é nulo ao nível de 5\% de significância, logo o modelo é multiplicativo. Para torná-lo aditivo e estabilizar a variância, foi fundamental efetuar uma transformação Box-Cox na escala dos dados. O valor de $\lambda$ estimado foi de 0,0864 e a transformação dos dados ocorreu de acordo com a equação 1 . 
Figura 2: Gráfico da amplitude versus média para subgrupos da série temporal de energia elétrica gerada pela matriz eólica.

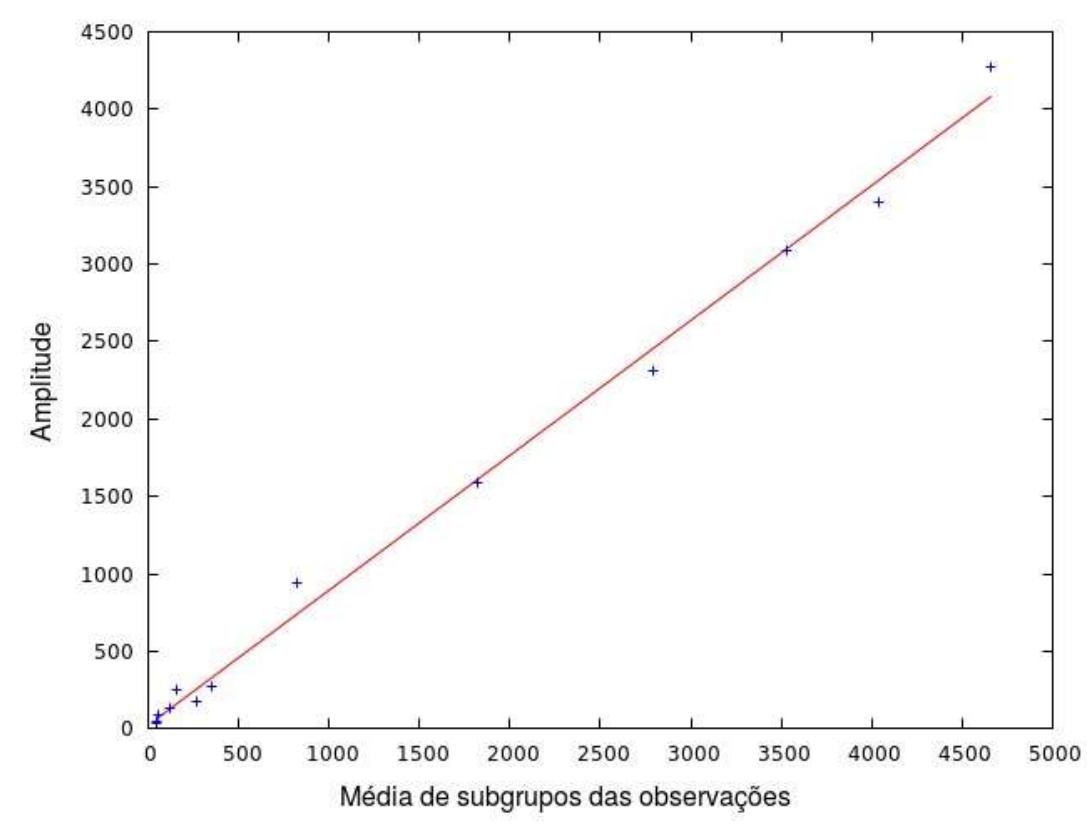

Fonte: Autores.

Segundo Ferreira et al. (2020), a existência de uma relação linear crescente ou decrescente entre os pontos do gráfico da amplitude em função da média indica necessidade de se realizar uma transformação nos dados.

Nota-se pelo correlograma da função de autocorrelação (FAC) da Figura 3 que a série temporal transformada não é estacionária, pois as defasagens decaem lentamente para zero. Na função de autocorrelação parcial (FACP) observam-se correlações maiores nas defasagens 1 e 3, com uma propensão para zero nas defasagens seguintes, evidenciando um provável processo de médias móveis.

Através do teste de Cox-Stuart, verificou-se a existência da componente de tendência com um p-valor de 0,01, rejeitando, ao nível de 5\% de significância, a hipótese nula de não existência de tendência. Dessa forma, foi preciso realizar uma diferença de ordem 1 na série transformada com o intuito de retirar o efeito da tendência.

O teste de Kruskal-Wallis foi aplicado e o resultado do p-valor foi de 0,7633, não rejeitando, ao nível de 5\% de significância, a hipótese nula de ausência de sazonalidade determinística, isto é, a série não necessita de uma diferença que considere a ordem de variação no período s. 
Figura 3: Correlograma das funções de autocorrelação (FAC) e autocorrelação parcial (FACP) da série temporal transformada.
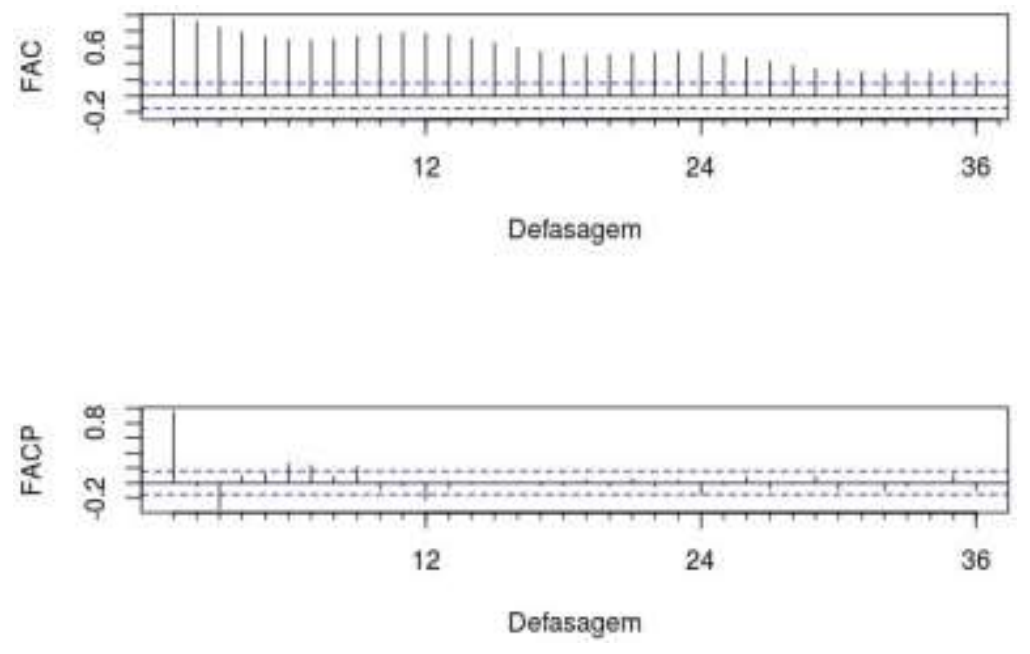

Fonte: Autores.

A Figura 4 apresenta os correlogramas da FAC e FACP para a série temporal transformada com uma diferença de ordem 1. A partir do correlograma da FACP, pode-se perceber a autocorrelação significamente diferente de zero nas defasagens 2, 3, 4, 5 e 6, indicando as possíveis ordens do modelo para a parte autorregressiva "p". Como não existem defasagens significativas múltiplas de 12 na FACP, a ordem sazonal de "P" é zero. No correlograma da FAC, as defasagens significativas são $2,3,5,6,7,8,11,12,13,16,17,18,19,23,24,25$ e 35, indicando as possíveis ordens do modelo para a parte de médias móveis "q". Como apareceram defasagens múltiplas de 12 significativas na FAC, a provável ordem sazonal de "Q" é dois. Assim, foi possível propor vários modelos SARIMA (p,d,q)×(P,D,Q)s.

Figura 4: Correlograma das funções de autocorrelação (FAC) e autocorrelação parcial (FACP) da série temporal transformada com uma diferença de ordem 1.
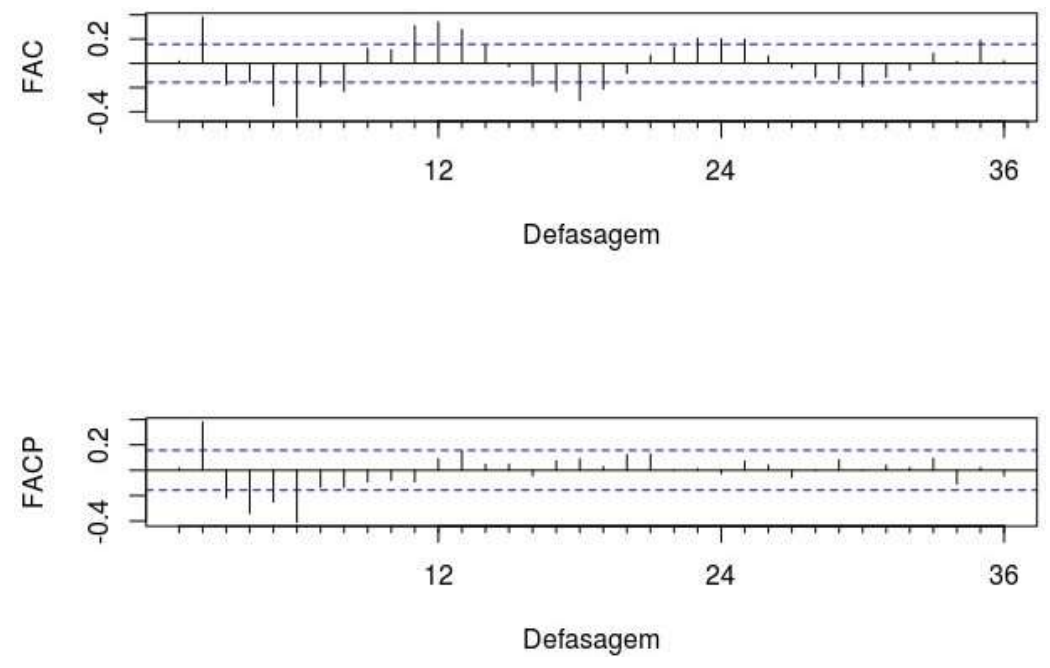

Fonte: Autores.

Dentre os modelos ajustados, buscaram-se aqueles com comportamento de ruído branco nos resíduos, isto é, apresentaram o p-valor superior a 5\% para o teste de Ljung-Box. Destes, selecionaram aqueles que tiveram menor critério de informação de Akaike (AIC), levando-se em conta o princípio da parcimônia, ou seja, menor número de parâmetros.

Na Tabela 1 estão os modelos com resíduos não correlacionados e com menor AIC, utilizados para realizar as 
previsões no período de jan/2020 a mar/21. A partir dessas previsões e das observações reais neste período, foi efetuado o cálculo do erro quadrático médio de previsão EQMP. Como um dos objetivos deste trabalho é realizar previsões para a produção de energia elétrica gerada pela matriz eólica, o modelo SARIMA $(5,1,2) \times(0,0,3)_{12}$ foi escolhido, pois apresentou o menor valor para a raiz quadrada do erro quadrático médio de previsão.

Tabela 1: Modelos SARIMA usados na previsão para validação dos dados através do critério de informação de Akaike (AIC) e da raiz quadrada do erro quadrático médio de previsão (EQMP).

\begin{tabular}{ccc}
\hline SARIMA (s=12) & AIC & لEQMP \\
\hline$(5,1,2) \times(0,0,2)$ & 97,47 & 773,79 \\
$(5,1,2) \times(0,0,1)$ & 96,71 & 788,18 \\
$(4,1,2) \times(0,0,1)$ & 94,78 & 794,87 \\
$(5,1,2) \times(1,0,1)$ & 96,83 & 787,18 \\
$(5,1,2) \times(0,0,3)$ & 99,26 & 765,61 \\
$(3,1,1) \times(0,1,1)$ & 103,17 & 1290,26 \\
\hline
\end{tabular}

Fonte: Autores.

Seguiu-se então para o ajuste de um modelo com todas as observações da série, desde jan/2007 a mar/2021, com o intuito de se fazer as previsões futuras da produção de energia elétrica gerada pela matriz eólica no Brasil no horizonte de abr/2021 a dez/2022. A metodologia utilizada para obtenção do primeiro ajuste foi realizada novamente passo a passo para se obter este novo ajuste. Mesmo com a inclusão das 15 observações (jan/20 a mar/21) ao processo de modelagem, o modelo SARIMA $(5,1,2) \times(0,0,3)_{12}$ continuou sendo o melhor ajuste.

A Tabela 2 mostra as estimativas dos parâmetros do modelo ajustado para descrever a série temporal, obtidas por meio do método de máxima verossimilhança e os seus respectivos erros padrão.

Tabela 2: Estimativas dos parâmetros do modelo SARIMA $(5,1,2) \times(0,0,3)_{12}$ ajustado para descrever a produção de energia elétrica gerada pela matriz eólica no período de jan/2007 a mar/2021.

\begin{tabular}{ccc}
\hline Parâmetro & Coeficiente & $\begin{array}{c}\text { Erro } \\
\text { padrão }\end{array}$ \\
\hline$\phi_{1}$ & 1,3837 & 0,0839 \\
$\phi_{2}$ & $-0,5408$ & 0,1383 \\
$\phi_{3}$ & $-0,1543$ & 0,1438 \\
$\phi_{4}$ & $-0,0630$ & 0,1407 \\
$\phi_{5}$ & $-0,0394$ & 0,0863 \\
$\theta_{1}$ & $-1,7091$ & 0,0329 \\
$\theta_{2}$ & 0,9997 & 0,0347 \\
$\Theta_{1}$ & 0,1833 & 0,0857 \\
$\theta_{2}$ & 0,1052 & 0,0908 \\
$\Theta_{3}$ & 0,0439 & 0,0967 \\
\hline
\end{tabular}

Fonte: Autores.

A Figura 5 ilustra a série histórica da produção de energia elétrica gerada pela matriz eólica no Brasil (em preto), o ajuste do modelo aos dados (em vermelho), a previsão para os meses de abr/2021 a dez/2022 (azul escuro) e os intervalos com 95\% de confiança para os meses previstos (azul claro). Nota-se que os valores ajustados ficaram próximos dos reais e que nos meses subsequentes previstos (em azul escuro) a série continua seguindo o padrão crescente que ela vem mantendo desde de 2015. 
Figura 5: Valores reais (preto), ajustados (vermelho), previstos (azul escuro) e intervalo com $95 \%$ de confiança (azul claro) para a produção de energia elétrica gerada pela matriz eólica abrangendo o período de jan/2007 a dez/2022.

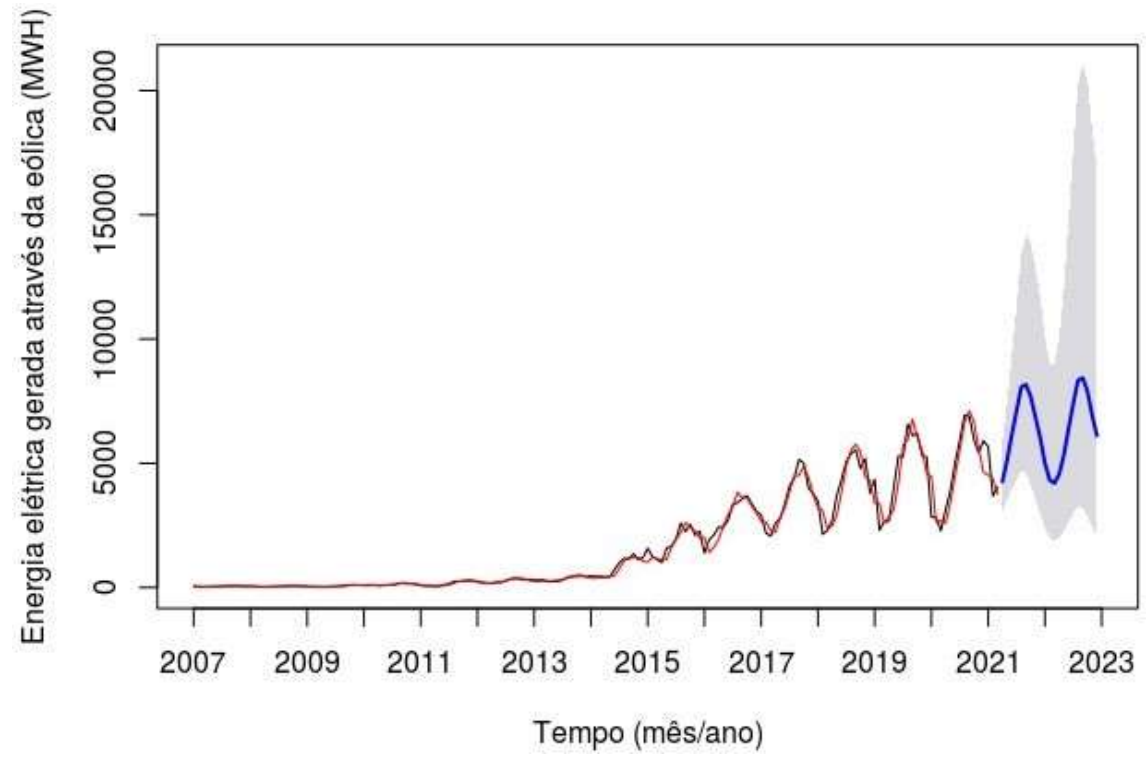

Fonte: Autores.

Constatou-se pela Figura 6 que todas as defasagens foram não significativas, corroborando com o resultado obtido pelo teste de Ljung-Box indicando que os resíduos não são correlacionados.

Figura 6: Correlograma da função de autocorrelação FAC residual do modelo SARIMA $(5,1,2) \times(0,0,3)_{12}$.

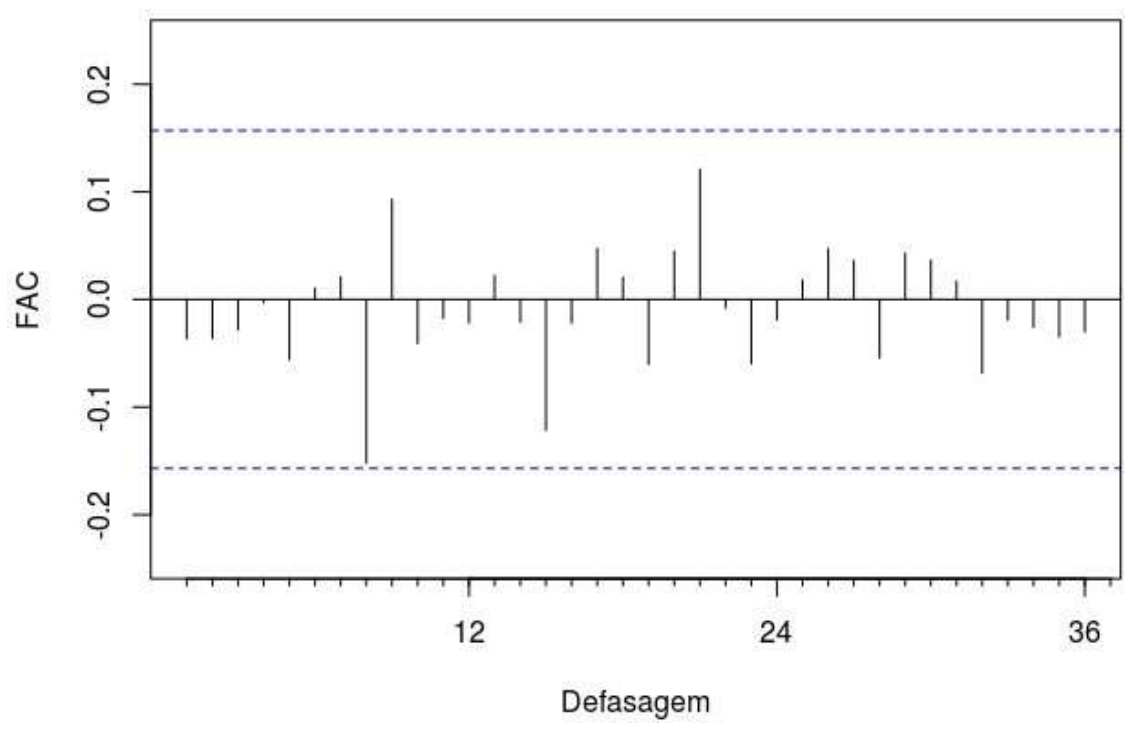

Fonte: Autores.

A Tabela 3 exibe os valores previstos e os respectivos intervalos com 95\% de confiança para a produção de energia elétrica gerada pela matriz eólica entre o período de abr/2021 a dez/2022. 
Tabela 3: Previsão da produção de energia elétrica gerada pela matriz eólica no período de abr/2021 a dez/2022.

\begin{tabular}{cccc}
\hline Meses & Limite inferior (2,5\%) & Previsto & Limite superior $(97,5 \%)$ \\
\hline Abril & 3085 & 4259 & 5841 \\
Maio & 3498 & 5126 & 7444 \\
Junho & 3981 & 6149 & 9388 \\
Julho & 4389 & 7103 & 11331 \\
Agosto & 4759 & 8071 & 13453 \\
Setembro & 4607 & 8170 & 14199 \\
Outubro & 4146 & 7676 & 13883 \\
Novembro & 3548 & 6844 & 12857 \\
Dezembro & 3005 & 6023 & 11720 \\
Janeiro & 2402 & 5003 & 10086 \\
Fevereiro & 2003 & 4323 & 9001 \\
Março & 1889 & 4199 & 8978 \\
Abril & 1979 & 4555 & 10050 \\
Maio & 2251 & 5321 & 12026 \\
Junho & 2636 & 6391 & 14774 \\
Julho & 2976 & 7411 & 17551 \\
Agosto & 3255 & 8337 & 20244 \\
Setembro & 3186 & 8434 & 21086 \\
Outubro & 2875 & 7888 & 20353 \\
Novembro & 2426 & 6912 & 18442 \\
Dezembro & 2070 & 6109 & 16813 \\
\hline
\end{tabular}

Fonte: Autores.

\section{Conclusão}

Os objetivos deste trabalho foram alcançados, pois conseguiu-se provar através de testes estatísticos que a série de produção de energia elétrica gerada pela matriz eólica possui uma componente de tendência, demonstrando que o Brasil vem se expandindo cada vez mais ao longo dos últimos anos. Observou-se que a série em questão não apresentou sazonalidade determinística. A variação sazonal encontrada é devida a volatilidade do vento, isso nos mostra que o Brasil possui uma incidência de ventos que pode ser aproveitada o ano inteiro, excluindo um ou dois meses (agosto e setembro) onde a temporada de ventos pode ser maior, conhecida como safra dos ventos, esse cenário contribui para fazer do país uma eficiente fonte de energia verde.

O modelo SARIMA $(5,1,2) \times(0,0,3)_{12}$ se adequou bem aos dados para prever a produção de energia elétrica gerada pela matriz eólica, baseando-se nos critérios de AIC e EQMP. Ele se mostrou bem eficiente, pois conseguiu captar a variabilidade da série. A modelagem forneceu previsões que revelam a ascensão desse tipo de energia renovável.

O modelo proposto para prever a quantidade de energia elétrica gerada pela matriz eólica de curto prazo vai permitir que os gestores tenham tempo suficiente para programar a operação de distribuição de energia de forma adequada.

Para trabalhos futuros, recomenda-se a atualização dos dados de energia eólica e a incorporação da análise de intervenção ao modelo SARIMA ajustado a fim de contribuir para uma previsão mais eficiente.

\section{Agradecimentos}

Os autores Mateus dos Santos Silva e Pedro Henrique Alves Bittecnourt Santos agradecem ao Programa Institucional de Iniciação Científica Voluntária PICV do CEFET-MG.

\section{Referências}

Alencar, D. B. (2018). Modelo híbrido baseado em séries temporais e redes neurais para prevenção da geração de energia eólica. Tese de doutorado, Universidade Federal do Pará, Belém, PA, Brasil.

Akaike, H. (1973). Maximum likelehood identification of gaussian autoregressive moving average models. Biometrika, $21,243-7$.

Box, G. E. P., \& Jenkins, G. M. (1976). Time Series Analysis: Forecasting and Control. Holden-Day. 
Braz, C. A., Rodrigues, R. L., \& Siqueira, H. V. (2017). Geração de energia elétrica por meio de fontes de energia renováveis: uma revisão sistemática. Revista de Gestão Industrial, 13(1), 228-42.

Delfino, O. A. S., Santos, P. H. A. B., \& Santos, R. V. R. (2021). Análise do comportamento da vazão de água na usina hidrelétrica do Funil/MG para realizar previsões e auxiliar na tomada de decisões. Sigmae, 10(1), 34-46.

Fernandes, L. C. (2018). Previsão de potência eólica de curtíssimo prazo baseada na análise espectral e decomposição da série temporal. Dissertação de mestrado, Universidade Federal de Pernambuco, Recife, PE, Brasil.

Ferreira, R. A., Miranda, V. F. L., Santos, P. M., \& Sáfadi, T. (2020). Um estudo sobre a evolução de óbitos por câncer de mama no Brasil usando modelos de séries temporais. Research, Society and Development, 9(12), 1-20.

Hyndman, R., Athanasopoulos, G., Bergmeir, C., Caceres, G., Chhay, L., O’hara-Wild, M., Petropoulos, F., Razbash, S., Wang, E., \& Yasmeen, F. (2019). Forecast: Forecasting functions for time series and linear models. R package version 8.5 , https://pkg.robjhyndman.com/forecast/

Lima, J. E. C., Castro, L. F., \& Cartaxo, G. A. A. (2019). Aplicação do Modelo Sarima na Previsão de Demanda no Setor Calçadista. Rev. Mult. Psic., 13(46), 892-913.

Lima, D. K. S., Leão, R. P. S., Santos, A. C. S., Melo, F. D. C., \& Chaves, C. M. N. M. (2013). Análise do potencial eólico offshore do Ceará em períodos de El Niño e La Niña. Revista Ciência e Natura, v. esp., 37-9.

Ljung, G. M., \& Box, G. E. P. (1978). On a measure of lack of fit in time series models. Biometrika, 65(2), 97-303.

Melo, E. (2013). Fonte eólica de energia: aspectos de inserção, tecnologia e competitividade. Estudos Avançados, 27(77), 125-42.

Malta, C. S. (2009). Estudos de séries temporais de vento utilizando análises estatísticas e agrupamento de dados. Trabalho de conclusão de curso, Universidade Federal do Rio de Janeiro, Rio de Janeiro, RJ, Brasil.

Martins, F. R., Guarnieri, R. A., \& Pereira, E. B. (2008). O aproveitamento da energia eólica. Revista Brasileira de Ensino de Física, 30(1), 1304-13.

Ministério de Minas e Energia. MME (2021). https://static.poder360.com.br/2021/08/1-Boletim-Mensal-de-Energia-Maio-2021-Portugues.pdf. Acesso em 10 de setembro de 2021 .

Morais, L. C. (2015). Estudo sobre o panorama da energia elétrica no Brasil e tendências futuras. Dissertação de mestrado, Universidade Estadual Paulista, Bauru, SP, Brasil.

Morettin, P. A., \& Toloi, C. M. C. (2006). Análise de Séries Temporais (2a ed.). Edgard Blucher.

Operador Nacional do Sistema Elétrico. ONS (2021). http://www.ons.org.br/Paginas/resultados-da-operacao/historico-da-operacao/geracao_energia.aspx.

Operador Nacional do Sistema Elétrico. ONS (2014). http://antigo.mme.gov.br/documents/239673/907477/Boletim_de_Monitoramento_do_Sistema_ Elxtrico_-_Fevereiro-2014.pdf/a52de0a5-aeee-bb66-05e8-7c7adc5862e6.

Pinto, R. J., \& Santos, V. M. L. (2019). Energia eólica no Brasil: evolução, desafios e perspectivas. Journal on Innovation and Sustainability, 10(1), 124-42.

Pereira, A. S., Shitsuka, D. M., Parreira, F. J., \& Shitsuka R. (2018). Metodologia da pesquisa científica. UFSM. https://repositorio.ufsm.br/bitstream/handle/1/15824/Lic_Computacao_Metodologia-Pesquisa-Cientifica.pdf?sequence=1

R Core Team. (2021). R: a language and environment for statistical computing. Vienna: R Foundation for statistical computing. http://www.Rproject.org.

Rodrigues, L. B., Perobelli, F. F. C., \& Vasconcelos, S. (2017). Geração de energia eólica no Brasil: um investimento viável. Revista Brasileira de Economia de Empresas, 17(2), 71-94.

Sáfadi, T. (2004). Uso de séries temporais na análise de vazão de água na represa de Furnas. Ciência e Agrotecnologia, 28(1), 142-8.

Salino, P. J. (2011). Energia eólica no Brasil: uma comparação do PROINFA e dos novos leilões. Trabalho de conclusão de curso. Universidade Federal do Rio de Janeiro, Rio de Janeiro, RJ, Brasil.

Santos, P. H. A. B., Delfino, O. A. S., Santos, R. V. R., \& Nascimento, M. (2021). Ajuste de um modelo de séries temporais para prever a precipitação pluviométrica. Research, Society and Development, 10(6), 1-11.

Shapiro, S. S., \& Wilk, M. B. (1965). An analysis of variance test for normality (complete samples). Biometrika, 52, 591-611.

Silva, H. B. (2018). Técnicas para redução de dimensionalidade de séries temporais e detecção de velocidades extremas do vento para geração de energia eólica. Dissertação de mestrado, Universidade Federal de Pernambuco, Recife, PE, Brasil.

Silva, P. K. O. (2017). Análise e previsão de curto prazo do vento através de modelagem estatística em áreas de potencial eólico no Nordeste do Brasil. Tese de doutorado, Universidade Federal de Campina Grande, Campina Grande, PB, Brasil.

Trapletti, A., \& Hornik, K. (2021). tseries: Time Series Analysis and Computational Finance. R package version 0.10-49, https://CRAN.Rproject.org/package=tseries. 